\title{
Testing of Event Detection Lateness in Wireless Sensor Networks
}

\author{
Laye Hadji Diakite ${ }^{1}, \mathrm{Li} \mathrm{Yu}^{1} \&$ Rabiu Haruna ${ }^{1}$ \\ ${ }^{1}$ Huazhong University of Science and Technology, Wuhan, China \\ Correspondence: Laye Hadji Diakite, Huazhong University of Science and Technology, Wuhan, China. E-mail: \\ hadjidiak@gmail.com
}

Received: July 27, 2013 Accepted: August 29, 2013 Online Published: September 28, 2013

doi:10.5539/cis.v6n4p103

URL: http://dx.doi.org/10.5539/cis.v6n4p103

\begin{abstract}
A wireless sensor network (WSNs) technology determines physical properties of the environment through detection of unusual events. The sensor consists of small mini computers powered by batteries and use radio communication for the exchange of information it gathers. The sensors are able to collect the data and exchange the information with all the nodes that were affected and triggered during the event detection. Although the technology has been in use for a period of time it has been noted that there is lateness of the event detection by the technology. Therefore the essence of this paper is to describe the factors affecting the lateness detection of events by WSNs. From the experiment it is evident that varying distance of the sensor nodes to the base station and the transmission power affect the packet data transmission efficiency and thus lateness detection of events. Power level at $5 \mathrm{dBm}$ (maximum level) ensures $90 \%$ of the event detected is communicated effectively to the base station whereas minimum power level at $-10 \mathrm{dBm}$ allows ineffective detection of events. At $-10 \mathrm{dBm}$ the base station node could not detect a transmitted data packet at distances beyond 30 feet. Thus the application of any sensor node with the transmitting power at $-10 \mathrm{dBm}$ will only be effective at the distance less than $10 \mathrm{ft}$. Therefore, distance of the sensor nodes and the transmission power of the WSNs affect the data packet delivery performance and hence lateness of event detection.
\end{abstract}

Keywords: wireless sensor networks, lateness event detection, event detection

\section{Introduction}

A wireless sensor network (WSNs) is a technology which has been in use for a period of time to determine physical properties of the environment. The wireless sensor network consists of small mini computers which are powered by batteries and use radio communication for the exchange of information it gathers from different sensors. The design enables the wireless sensor networks to detect any unusual event as long as they can because the batteries are able to store energy for a long period of time. The sensors are diverse and they are able to detect any event of interest. They also collect data and exchange the information with all the nodes that were affected and triggered during the event detection.

WSNs is able to detect events in its field of application by gathering the raw data from one to thousands nodes or sensors connected to it, recognizing what WSNs did previously and mapping the event and systematically transmit the event into the communication unit or central based station. WSN can either employ the simple technique or special pattern of algorithm recognition or go for the complex hardware for recognition in order to ensure proper event detections are transmitted from the stationed sensors to the central based station.

Detection of event by the WSNs requires that the system should distinguish between the presence and the absence of any unusual event in the environment. Successful detection of the events requires that each node of the sensor transmits the any detection precisely and accurate to the central based station. The key performance of the WSNs is determined through the maximum probability of correct detection and transmission of the results. Any false transmission or late detection of the event to the central based station leads to inappropriate communication between the networks.

The essence of this paper is to bring out the challenges which the WSNs technology faces when it comes to detection and bring out the main setbacks that bring differences in the event detection in different fields where WSNs is used. This paper is different from other papers because it is able to indicate the differences in lateness in event detection while other papers have been able to focus on the different applications of WSNs in different fields and the strengths of one technology over the other. 


\subsection{Research Questions}

- Does distance of the sensor nodes affect the lateness detection of the event by WSNs technology?

- Does the transmitting power of the WSNs affect the data packet delivery performance and hence lateness of event detection?

\subsection{Hypothesis}

Hypothesis 1

Null hypothesis: Distance of the sensor node does not have any significant impact on the lateness detection of the event by WSNs.

Alternative hypothesis: Distance of the sensor significantly affects the lateness detection of the event by WSNs.

Hypothesis 2

Null hypothesis: Transmitting power has no significant relationship with the data packet delivery performance of the WSNs.

Alternative hypothesis: Transmitting power has a significant relationship with the data packet delivery performance of the WSNs.

\section{Literature Review}

According to Pottie and Kaiser (Pottie \& Kaiser, 2000), WSNs technology should be able to manage power at all levels so that to ensure that the functionality and hierarchy of signals of the unusual events are taken as they occur so that to reduce the cost of communication between different nodes of the WSNs technology.

Sagatov, Sukhov and Calyam (2010) highlighted that the quality of the wireless networks is affected when the packet loss is approximately $80 \%$ and when the network jitter is about $20 \%$. Netstumbler is very important to detect causes of wireless interference, unauthorized events due to access points and any other unusual event (Netstumbler).

Kahn, Katz, and Pister (1999) explained that detection differences among the WSNs machines are present and mostly caused by the networks of millimeter-scale system whereby there are differences in communications. They were able to show that the differences in detection of events are as a result of the bit rate, energy per bit and energy per bit during the transmission of the unusual events in the WSN.

According to Seyba, Mullins and Bonafede (2007), in their study of audio-video capacity, they indicated that streaming of the video and audio using the wireless networks using Wi-Fi, GSM, 3G, which is an old standard is very hard due to large percentage of packet loss. They indicated that when the percentage of packet loss exceeds $0.5 \%$ the quality of wireless network will not be good. They went further and indicated that there will be no streaming when the percentage is over $1.5 \%$.

According to Tennenhouse (2000), he suggested that it is important for the designers of the WSNs systems to take into consideration the number of networks to ensure efficient transmission of the waves and information during communication from the thousands nodes connected to each machine. He specified that the machines should be able to ensure that the real and physical information about the unusual event are recorded and transmitted as they occur in the different places where WSNs are applied. According to him it is important to have technique which is simple and friendly and able to get real information which is relevant to the event detection.

WSNs technology should be able to detect random events occurring at unpredictable frequencies, time and points where they are applied. This technology detects events through the application of statistical analysis which is able to describe many events occurring in a period of time. Poisson distribution is mostly employed in these strategies to calculate the number of events occurring in the period of time because they occur frequently. The events might occur randomly in a given period of time, either in seconds, minutes, days and even years. The frequency can be spatial or temporal depending on the events detected within a specified period of time. The technology employs poison distribution techniques which possess inter-events times that are usually independent, jointly, identical and follow a certain exponential probability.

The Poisson distribution probability of a random variable A i.e. the number of events occurring in a given period of time can be easily detected by the WSNs in a specified place or region through the following formula:

$$
\mathrm{P}(\mathrm{x} ; \mu)=\frac{\mathrm{e}^{-\mu} \mu^{\mathrm{x}}}{\mathrm{x} !}, \mathrm{x} \in\{0,1,2,3 \ldots\}
$$


where $\mu$, represents the average number of events WSNs is able to detect in a given period of time in a specified region (Walpoke, 1972).

Therefore the application of WSNs should be able to follow Poisson probability distribution. When the technology is not able to follow the above Poisson probability distribution there will be a failure in the system and the information collected or detected will have errors and it will not be true. This specifically occurs with rare or rarely events which the sensor might not be able to figure may be missed out thus causing an error. With that the researcher will be able to detect in delay using the Poisson probability.

According to a Poisson probability to monitor an event, nodes are considered to be located randomly where $\mathrm{p}$ is the node density. The definition of n-delay of an event is the delay between when the event occurs and when the event is $n$-detected. The (n-p) delay bound of an event is time in which the event is $n$-detected with probability $p$. Through these definitions the researcher can be able to determine the n-delay distribution of an event, average delay of $n$-event and the (n-p)-delay bound of an event.

\section{Matlab Experiment}

\subsection{Data Transmission}

For the data to be transmitted to the central or base station it depends with the level to which the nodes of the various sensors are lying as compared to the base station. If the sensor node is lying within the range of the base station then data transmission will be direct and if the node is far away from the base station then data will be transmitted through diagonal routing. Thus the distance matters a lot when it comes to data transmission.

\subsection{Energy Consumption}

WSNs sensors use power to detect unusual events and transmission of data to the central station. For one to understand the amount of power the technology uses for transmitting and receiving the data he applies the following equation:

$$
\begin{gathered}
\mathrm{P}_{\mathrm{T}}(\mathrm{d})=\mathrm{P}_{\mathrm{TB}}+\mathrm{P}_{\mathrm{TRF}}+\mathrm{P}_{\mathrm{A}}(\mathrm{d})=\mathrm{P}_{\mathrm{T} 0}+\mathrm{P}_{\mathrm{A}}(\mathrm{d}) \\
\mathrm{P}_{\mathrm{R}}=\mathrm{P}_{\mathrm{RB}}+\mathrm{P}_{\mathrm{RRF}}+\mathrm{P}_{\mathrm{L}}=\mathrm{P}_{\mathrm{R} 0}
\end{gathered}
$$

Where PT, denotes the transmitting power and the PR is the receiving power. PA(d) is the Amplifiers power consumption, and $\mathrm{d}$ is the range of the transmission. PTB refers to the baseband power consumption during transmission and PRB is the power consumption in baseband of receiving. PTRF is the power consumption in front-end circuit for transmitting and PRRF is refers to the front-end circuit power for receiving and PL is the power consumption of LNA for receiving. For the receiving end a constant PR0, can also be used to represent the PRB, PRRF and PL. The total power consumption of the amplifier, PA(d), depends on the specification the hardware, loading characteristics, operating frequency and the power output of the WSNs machine (Lee, 2004).

\subsection{Throughput}

This refers to the average rate in which WSNs sensors are able to successfully delivered packets over a certain network. It is usually measured in data packets per second. WSNs event detection capabilities can be improved by making sure that nodes or the sensors placed at different positions are able to send the data directly to the base station rather than to send them through the diagonal routing.

\subsection{Experimental Results}

For the study to achieve its objectives two experiments were conducted to determine the lateness detection of the event by the machine in a given field of application. The first experiment was conducted to determine the data packet delivery performance from the different sensors placed at different locations to the base station and communication center.

Another experiments demonstrated how the transmitted power of the sensor node affects the delivery of the packet data to the communication system. It is important to note that delivery performance of the data determines the overall performance of the WSNs technology in its field of application.

\subsubsection{Distance and the Varying Transmitting Power of the Sensor Nodes}

This experiment was conducted by locating different sensor nodes at different locations from the base station. Each sensor node was expected to detect the environmental event and transmit the data to the base station. The power in each node was set at different values to determine if there is any effect of power on the transmission of data. The sensor node was expected to transmit data at the rate of 1 packet per second.

The transmission power of the experiment was configured at different levels to allow the researcher to determine 
if there is any effect of power on the lateness of event detection by the WSNs. The power was put at the maximum level of $5 \mathrm{dBm}$ and each sensor within a particular distance was supposed to detect an event and send the data to the base station. The experiment was repeated with the power level at $0 \mathrm{dBm}$ (intermediate level) and another one at $-10 \mathrm{dBm}$ (lowest level) respectively. The experiment was done several times using different power levels and distance of the sensor node. The result of the experiments was recorded in a table as below.

Table 1. Results of the experiment using different power levels and distance of the sensor node

\begin{tabular}{cccc}
\hline Distance in foot & $\begin{array}{c}\text { Packet data acceptance } \\
(\%) \text { at } 5 \mathrm{dBm}\end{array}$ & $\begin{array}{r}\text { Packet data acceptance } \\
(\%) \text { at } 0 \mathrm{dBm}\end{array}$ & $\begin{array}{c}\text { Packet data acceptance } \\
(\%) \text { at }-10 \mathrm{dBm}\end{array}$ \\
\hline 1 & 99.1 & 95.8 & 82.5 \\
2 & 96.2 & 95.5 & 77.8 \\
3 & 97.1 & 94.8 & 73.1 \\
4 & 94.0 & 95.1 & 72.3 \\
5 & 93.2 & 91.6 & 69.1 \\
10 & 94.1 & 93.7 & 55.7 \\
15 & 94.2 & 77.2 & 0.8 \\
20 & 94.4 & 86.3 & 0.5 \\
25 & 93.8 & 78.8 & 0.3 \\
30 & 92.1 & 75.0 & 0.0 \\
\hline
\end{tabular}

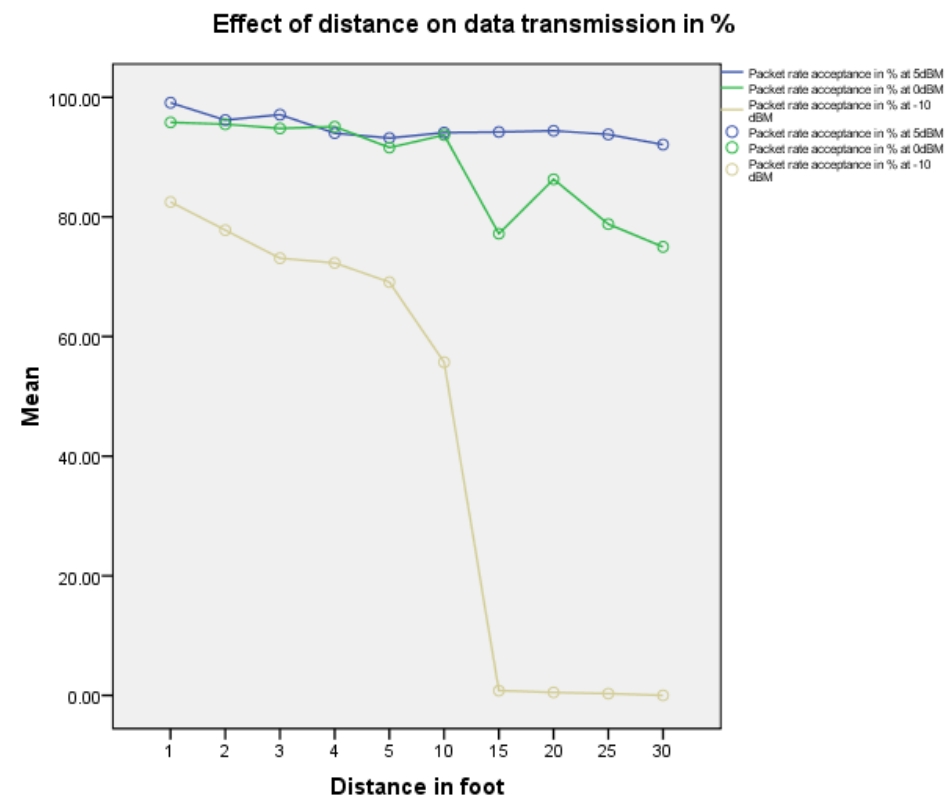

Figure 1. Line graph showing the data packet delivery performance with varying distance and power of the WSNs sensor nodes

From the above line chart it is evident that distance and power affects the delivery of the packet data and hence the performance of the WSNs machine. This is evident from the figure because $90 \%$ and above of the data packet delivery was achieved with the power of the sensor nodes at the maximum level $(5 \mathrm{dBm})$ no matter the distance of the sensor node. When the power amplifier is at $0 \mathrm{dBm}$, the event sensor node was able to obtain a PAR of $90 \%$ or more for distances within a 10 -foot radius. For distances beyond 10 feet the delivery of packet data ranged from $86.3 \%$ to $75 \%$. At $-10 \mathrm{dBm}$, the event sensor was unable to obtain delivery performance of $90 \%$. The base station node could not detect a transmitted data packet at distances beyond 30 feet. Thus the application of any sensor node with the transmitting power at $-10 \mathrm{dBm}$ will only be effective at the distance less 
than $10 \mathrm{ft}$. The data packet delivery performance varies with the distance of the sensor nodes and the power of the transmission. At 1 foot the variation differs with power transmission in the sense that the variation between the $5 \mathrm{dBm}$ and $0 \mathrm{dBm}$ is approximately $3.3 \%$ and that of $5 \mathrm{dBm}$ and $-10 \mathrm{dBm}$ is $16.6 \%$. The variation increases with distance and with the power of transmission and thus at lower power and increase in distance there would be weak detection and transmission of packet data. The results of this study is supported by Seyba et al. (2007) where they indicated that when the percentage of packet loss which exceeds $0.5 \%$ affects the quality of the wireless connection and streaming of video and audio. This suggests that the coverage range of any event sensor programmed at an output power level of $-10 \mathrm{dBm}$, would be 10 feet.

The impact of the communication hardware of the WSNs on total power consumption not only depends on the parameters of radio environment (i.e. $\varepsilon$ and $\alpha$ ), but also depends on the drain efficiency of the power amplifier.

Table 2. The strength of the event detection is also affected when the signal passes through the human body

\begin{tabular}{cc}
\hline Test number & Packet Acceptance Rate $\%$ \\
\hline 1 & $76 \%$ \\
2 & $82 \%$ \\
3 & $69 \%$ \\
4 & $75 \%$ \\
5 & $77 \%$ \\
6 & $68 \%$ \\
7 & $70 \%$ \\
\hline
\end{tabular}

From the above results it is evident that strength of the Packet Acceptance Rate is decreased drastically when the signal has to pass through the human body. The result shows that the range is from $68 \%$ to $82 \%$ which is usually lower than the Packet Acceptance Rate which is above $90 \%$ at $5 \mathrm{dDm}$ as indicated in the above experiment. Delays of the event detection varies as it is evident from this study that the variation occurs in the course of network interruption in the essence that when there are no interruption in the network the packet delivery is above $90 \%$ and when there is interruption the delivery performance decreases approximately by $10 \%$ and above. The result of this study goes hand in hand with Sagatov et al. (2010) where they highlighted that the quality of the wireless networks is affected when the packet loss is approximately $80 \%$ and when the network jitter is about $20 \%$. Therefore the structure of the human body affects the packet acceptance rate and therefore lateness detection of the events and communication among the network.

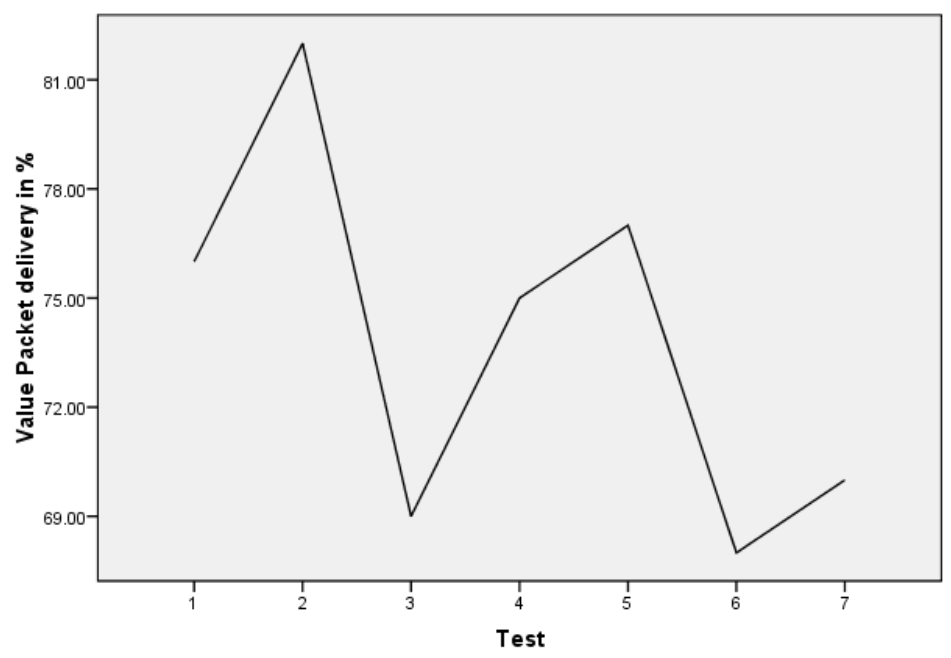

Figure 2. Value packet delivery 
The Figure 2 shows that the value of the ranges from $68 \%$ to $82 \%$ when it passes through the human body thus affecting the mean throughput of the wireless network sensors.

Table 3. Mean throughput

\begin{tabular}{ccc}
\hline Data packets & Mean throughput & Number of nodes \\
\hline 4 packets per second & 4.0 & 1 \\
4 packets per second & 3.0 & 2 \\
4 packets per second & 4.0 & 3 \\
4 packets per second & 5.0 & 4 \\
4 packets per second & 4.5 & 5 \\
10 packets per second & 10.0 & 1 \\
10 packets per second & 10.0 & 2 \\
10 packets per second & 9.0 & 3 \\
10 packets per second & 8.0 & 4 \\
10 packets per second & 7.0 & 5 \\
15 packets per second & 14.5 & 1 \\
15 packets per second & 14.5 & 2 \\
15 packets per second & 14.0 & 3 \\
15 packets per second & 13.0 & 4 \\
15 packets per second & 10.0 & 5 \\
\hline
\end{tabular}

From the above experiments it is evident that when mean throughput of the signals are send at 4 packets per second the signal will not change as the number of nodes is increased but at 15 packets per second the data transmission is affected when the nodes increase in number.

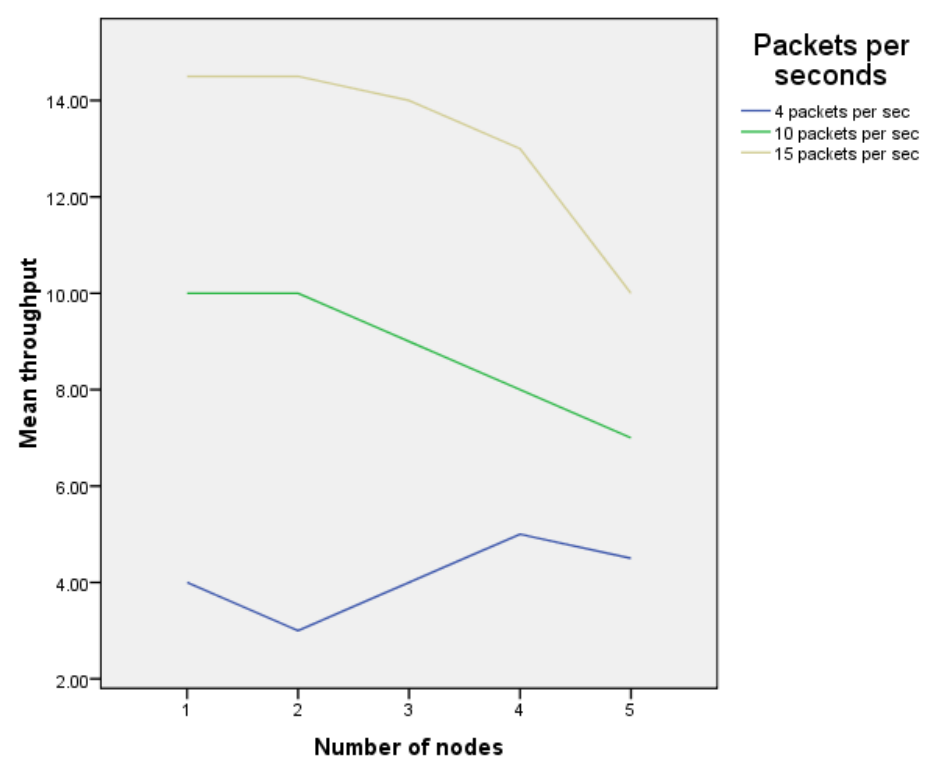

Figure 3. Mean throughput of data packets per number of nodes

From the above figure it is evident that mean throughput of the signals are send at 4 packets per second are consistent while that the 10 packets per sec and 15 packets per second decrease when the number of nodes 
increase.

\section{Conclusions}

We have been able to look at the various ways in which distance and varying power can affect the event detection of the WSNs. Lateness of the event detection is due to the above two factors. Distance affects the packet data delivery efficiency of the different sensors nodes placed at different distance thus affecting the performance of the WSNs technology. Sensor nodes near the sink get depleted thus creating gaps in the network which might affect the communication channel. Therefore it is evident that distance of the sensor nodes affects the lateness detection of the event by WSNs technology.

Transmission power has an effect on the sensor node data delivery efficiency and thus affecting the WSNs performance and event detection in general. Power level at $5 \mathrm{dBm}$ (maximum level) ensures $90 \%$ of the event detected communicated effectively to the base station whereas minimum power level at $-10 \mathrm{dBm}$ allows ineffective detection of events. The base station node could not detect a transmitted data packet at distances beyond 30 feet. Thus the application of any sensor node with the transmitting power at $-10 \mathrm{dBm}$ will only be effective at the distance less than $10 \mathrm{ft}$. Therefore transmission power of the WSNs affect the data packet delivery performance and hence lateness of event detection.

Therefore it is important for the manufacturers to come up with recommended distance and power level for which the WSNs machines can work with effectively without any lateness in event detection and loss of data on the way as transmission is done.

\section{References}

Kahn, J. M., Katz, R. H., \& Pister, K. S. (1999, August). Next century challenges: mobile networking for "Smart Dust". In Proceedings of the 5th annual ACM/IEEE international conference on Mobile computing and networking (pp. 271-278). ACM.

Lee, T. H. (2004). The design of CMOS radio-frequency integrated circuits. Cambridge university press.

NetStumbler. (2013). Wi-Fi Security - The Rise and Fall of WPS. Retrieved from http://www.netstumbler.com/

Pottie, G. J., \& Kaiser, W. J. (2000). Wireless integrated network sensors. Communications of the ACM, 43(5), 51-58. http://dx.doi.org/10.1145/332833.332838

Sagatov, E. S., Sukhov, A. M., \& Calyam, P. (2010, September). Influence of distortions of key frames on video transfer in wireless networks. In I/V Communications and Mobile Network (ISVC), 2010 5th International Symposium on (pp. 1-4). IEEE.

Seyba, J. R., Mullins, B. E., \& Bonafede, G. L. (2007, May). Audio-video capacity of an IEEE $802.11 \mathrm{~g}$ wireless LAN. In Collaborative Technologies and Systems, 2007. CTS 2007. International Symposium on (pp. 372-378). IEEE.

Tennenhouse, D. (2000). Proactive computing. Communications of the ACM, 43(5), 43-50. http://dx.doi.org/10.1145/332833.332837

Walpoke, R., \& Myers, E. (1972). Probability and Statistics for Engineers and Scientists. New York: MacMillan Publishing Co., Inc.

\section{Copyrights}

Copyright for this article is retained by the author(s), with first publication rights granted to the journal.

This is an open-access article distributed under the terms and conditions of the Creative Commons Attribution license (http://creativecommons.org/licenses/by/3.0/). 